\title{
Study of the Charge Dynamics in Mineral Oil under a Non-homogeneous Field
}

\author{
Yuan Zhou, Miao Hao, George Chen \\ Electronics and Electrical Engineering \\ University of Southampton \\ SO17 1BJ UK \\ Gordon Wilson and Paul Jarman \\ National Grid UK \\ Warwick, CV34 6DA, UK
}

\begin{abstract}
The presence of mobile charge carriers can affect the frequency response of complex permittivity of insulating liquid when the frequency is low. It has been found that the experimental results of the complex permittivity of mineral oils are in a good agreement with Coelho's frequency response theory when an extra contribution to the complex permittivity arising from DC conduction is taken into account. It has been reported that small quantity of high mobility charge carriers are responsible for this DC conduction. In our previous work, we have found that if the charge carriers are fast enough so that they can reach the opposite electrode in a cycle, the motion of these fast charge carriers can only contribute to the imaginary part of the complex permittivity under a homogeneous field. In this paper, the polarization induced by the motion of those fast charge carriers under a non- homogeneous field has been studied. It has been found when the field is not high, the total current caused by the drift and diffusion of those fast charge carriers is proportional to the external voltage. A modified space charge polarization theory has been proposed. The frequency dependence of the complex permittivity obtained experimentally has been fitted using this modified Coelho model and compared with our previous simulation. This modified model enables one to gain a better understanding of the frequency response in mineral oil in low frequency regions.
\end{abstract}

Index Terms - Oil aging, dielectric spectroscopy, polarization, charge carrier

\section{INTRODUCTION}

MINERAL oils, with the main roles of insulating and cooling, greatly affect the performance of high voltage transformers. Various diagnostic methods have been applied to monitor dielectric changes in insulating liquid over time, no matter these changes are due to thermal, electrical or chemical effects [1-4]. Dielectric spectroscopy technique, which enables us to gain a bunch of information about the dynamics of bound and mobile charge carriers, has been widely employed in the evaluation of insulating properties of dielectric liquids [4-18].

Mobile charges in dielectric media can form electrical double layers as a capacitor at the electrodes at low frequency and result in an anomalous increase in the real part of the complex permittivity [19]. This dielectric effect is known as space charge polarization and its influence on the complex permittivity becomes notable when the frequency is below several $\mathrm{kHz}$ [19]. The dielectric loss caused by the space charge polarization can be considerably large

Manuscript received on 12 November 2014, in final form 11 March 2015, accepted 28 March 2015. so that the contributions to the complex permittivity arising from electronic, atomic and dipole polarizations can be masked [19]. This space charge polarization has been studied both theoretically and experimentally by many researchers [20-37]. Jaffe proposed a theoretical solution of the space charge polarization and his theory has been used to analyse the dielectric response of electrolytic solutions and dielectric liquid [20-23]. Macdonald presented more general expressions and his theory has been widely accepted and used to analyse the experimental result [24-30]. Sawada simplified the polarization theory and proposed a computer based method to calculate the frequency response in liquids. [31-35]. Coelho assumed the mobile charges that move towards the electrode of opposite sign can accumulate in the vicinity of the electrode and result in a macro dipole finally [36]. Frood and Gallagher discovered that the experimental results of the frequency response of liquid are in agreement with Coelho's theory only when an additional contribution to the permittivity arising from DC conductivity has been taken into account [37]. It has been reported that if the polarization induced from DC conductivity is added to the total polarization, a better fit to the experimental results can be achieved [37-41]. 
In our previous work, a model has been proposed for oil dielectric response in frequency response with two types of charge carriers being involved [42-44]. The first kind of charge carriers is the normal ions in the mineral oil and they can be blocked by the electrodes, whilst the second kind is created in a region near the electrode and can be neutralized when they get close to the opposite electrode. The mobility of the second kind of charge carriers should be fast enough so that the polarization contributed by their motion is consistent with that arising from DC conduction [43]. Later on, a simple modified polarization theory has been proposed based on Coelho's work and this modified theory can well explain the dielectric behaviour in mineral oil in the frequency domain [36, 44]. If charge carriers are not evenly distributed between electrodes, the electric field will be distorted by the presence of space charge. A theory of space charge polarization with fast charge carriers as well as positive and negative ions presenting in mineral oil, to our knowledge, has not been developed. In this paper, a modified Coelho model under a non- homogeneous field will be developed. The frequency response calculated from this modified theory will be used to compare with the experimental results as well as our previous computer based simulation result.

\section{COELHO'S SPACE CHARGE POLARIZATION THEORY}

Before introducing the modified Coelho model, the polarization model developed by Coelho will be reviewed [36]. In his model, he assumed that there were parallel plate electrodes contain both positive and negative mobile charge carriers and the diffusion coefficient, mobility, density and charge carried by a single charge carriers of positive charge carriers were equal to those of negative charge carriers. In the absence of external voltage, these charge carriers are distributed evenly between electrodes. When a field is applied, these charge carriers are drifting towards electrodes. These charge carriers can be blocked by the electrode and form charge layers.

If $n_{+}(x, t)$ and $n_{-}(x, t)$ are the density of positive and negative charge carriers at $x$ at time $t$ and the charge carried by a single charge carrier is $q$, the net charge density can be denoted as:

$$
\rho(x, t)=q\left[n_{+}(x, t)-n_{-}(x, t)\right]
$$

When a field is applied, those charge carriers experience both influences from electric field and thermal diffusion. The current density is given by:

$$
j(x, t)=j_{+}(x, t)-j_{-}(x, t)
$$

with

$$
\begin{gathered}
j_{+}(x, t)=q n_{+}(x, t) \mu_{+} E(x, t)-D_{+} q \frac{\partial n_{+}(x, t)}{\partial t} \\
j_{-}(x, t)=-q n_{-}(x, t) \mu_{-} E(x, t)-D_{-} q \frac{\partial n_{-}(x, t)}{\partial t}
\end{gathered}
$$

where, $\mu_{+}, \mu_{-}, D_{+}$and $D_{-}$are the mobility and diffusion coefficients of the positive and negative charge carriers, respectively. After using Einstein's relationship, the diffusion coefficient can be described as

$$
D_{ \pm}=\frac{\mu_{ \pm} k T}{q}
$$

where, $k$ is the Boltzmann's constant, $T$ is the absolute temperature.

The equation of charge conservation are given by

$$
q \frac{\partial n_{ \pm}(x, t)}{\partial t}+\frac{\partial j_{ \pm}(x, t)}{\partial x}=0
$$

Noting that the electric field must be subject to Poisson equation

$$
\frac{\partial E(x, t)}{\partial x}=q\left[n_{+}(x, t)-n_{-}(x, t)\right] / \varepsilon_{0} \varepsilon_{r}
$$

where, $\varepsilon_{0}$ is the dielectric constant of vacuum and $\varepsilon_{r}$ is the relative dielectric constant of the liquid.

If $n_{+}(x, t)$ and $n_{-}(x, t)$ do not differ much from their equilibrium values, they can be linearized as

$$
\begin{aligned}
& n_{+}(x, t)=n_{0}+a(x, t), \\
& n_{-}(x, t)=n_{0}+b(x, t),
\end{aligned}
$$

where, $n_{0}$ is the equilibrium value of the density of positive or negative charge carriers and $a(x, t)$ and $b(x, t)$ are much smaller compared to $n_{0}$.

When the diffusion coefficient and mobility of positive charge carriers are equal to those of negative charge carriers, $D=D_{+}=D_{-}$and $\mu=\mu_{+}=\mu_{-}$, by substituting Eq. (7a) and (7b) into Eq. (2) and (5) we can obtain,

$$
\begin{gathered}
j(x, t)=\sigma E(x, t)-D \frac{\partial \rho(x, t)}{\partial x} \\
\frac{\partial \rho(x, t)}{\partial t}+\frac{\sigma}{\varepsilon_{0} \varepsilon_{r}} \rho(x, t)=D \frac{\partial^{2} \rho(x, t)}{\partial x^{2}}
\end{gathered}
$$

where, $\sigma$ is the liquid conductivity and $\sigma=2 n_{0} q \mu$. If we assume, in steady state, solution of Eq. (9) has the form:

$$
\rho(x, t)=\rho_{\omega}(x) e^{i \omega t}
$$

where $\omega$ is the angular frequency. The electric field and current density should also have the same form

$$
\begin{aligned}
& E(x, t)=E_{\omega}(x) e^{i \omega t} \\
& j(x, t)=j_{\omega}(x) e^{i \omega t}
\end{aligned}
$$

Coelho noted that those charge carriers could not get across the electrodes at $x= \pm d$, thus the current density at the two metal electrodes should follow

$$
\sigma E_{\omega}( \pm d)=D\left|\frac{\partial \rho_{\omega}}{\partial x}\right|_{(x= \pm d)}
$$

With this boundary condition, the charge density, field and current density can be calculated as:

$$
\begin{gathered}
\rho_{\omega}(x)=\frac{\varepsilon_{0} \varepsilon_{r} E_{a} \sinh \left(k_{\omega} x\right)}{M} \\
E_{\omega}(x)=E_{a} \frac{\cosh \left(k_{\omega} x\right)+i \omega \tau \cosh \left(k_{\omega} d\right)}{M}
\end{gathered}
$$




$$
j_{\omega}(x)=i \omega \tau \sigma E_{a} \frac{\cosh \left(k_{\omega} d\right)-\cosh \left(k_{\omega} x\right)}{M}
$$

with

$$
\begin{gathered}
\tau=\frac{\varepsilon_{0} \varepsilon_{r}}{\sigma} \\
k_{\omega}^{2}=\frac{(1+i \omega \tau)}{D \tau} \\
M=\frac{\sinh \left(k_{\omega} d\right)}{k_{\omega} d}+i \omega \tau \cosh \left(k_{\omega} d\right)
\end{gathered}
$$

where, $E_{a}$ is the magnitude of the electric field, $\tau$ is a constant related to the nature of the liquid, $k_{\omega}$ and $M$ are frequency dependent parameters.

Note that when $\omega=0, j_{0}(x)=0$, therefore, there is no DC conduction in Coelho's model.

The current density in the external circuit can be calculated using the following expression:

$$
\begin{aligned}
j_{\text {ext }}(\omega) & =i \omega\left(\varepsilon_{0} \varepsilon_{r}-\varepsilon_{0}\right) E_{a} \\
& +i \omega \tau \sigma E_{a} \frac{\cosh \left(k_{\omega} d\right)-\sinh \left(k_{\omega} d\right) /\left(k_{\omega} d\right)}{M}
\end{aligned}
$$

The first term of Eq. (20) is induced by the displacement current in the media, whilst the second term is induced by the motion of charge carriers. This current density must be equal to the current density in a sample that has a permittivity of $\varepsilon(\omega)$,

$$
j_{\text {ext }}(\omega)=i \omega\left(\varepsilon(\omega)-\varepsilon_{0}\right) E_{a}
$$

Comparing Eqs. (21) and (20) leads to the complex permittivity:

$$
\varepsilon(\omega)=\varepsilon_{0} \varepsilon_{r}\left[\frac{1+i \omega \tau}{i \omega \tau+\tanh \left(k_{\omega} d\right) / k_{\omega} d}\right]
$$

Equation (22) is Coelho's main result [36].

It is worth mentioning $\sigma, \varepsilon_{r}, D_{ \pm}$and $\mu_{ \pm}$are temperature dependent parameters. As the dissociation coefficient of mineral oil increases with temperature, more charge carriers will be generated through ionic dissociation, resulting in a high conductivity if the temperature rises. As reported by other researchers, the relative permittivity of insulting oil changes slightly with the temperature [4-18]. The viscosity of mineral oil decreases with temperature. When the temperature is higher, the charge carriers can move faster as the friction force acting upon them is reduced. Thus, the mobility of the charge carriers increases with temperature. As shown in equation (4), the diffusion coefficient is proportional to the mobility. If the temperature effect is considered, $\tau$ and $k_{\omega}$, defined in equations (17) and (18), are also temperature dependent. Consequently, equation (22) should be written as:

$$
\varepsilon(\omega)=\varepsilon_{0} \varepsilon_{r}(T)\left\{\frac{1+i \omega \tau(T)}{i \omega \tau(T)+\tanh \left[k_{\omega}(T) d\right] / k_{\omega}(T) d}\right\}
$$

\section{MODIFED POLARIZATION MODEL}

In our previous study, it was assumed there are two kinds of charge carriers in mineral oil. The first kind of charge carriers have a low mobility and can be blocked by the electrode to form the charge layers, whilst the second kind have a high mobility and can be charged at one electrode and discharged when they approach the electrode with the opposite sign [4244]. The polarization caused by the second kind of charge carriers under a homogeneous field has been studied [43]. Here, these two kinds of charge carriers are referred as the fast charge carriers and slow charge carriers, respectively.

The current contributed by the drift of these fast charge carriers under a homogeneous field has been theoretically studied and the polarization induced by the motion of those fast charge carriers can be given as [43]

$$
\left\{\begin{array}{l}
\Delta \varepsilon^{\prime}(\omega)=0 \\
\Delta \varepsilon^{\prime \prime}(\omega)=\frac{q_{i}^{0} \mu_{i}}{\omega \varepsilon_{0}} \times \frac{2 \mu_{i} V_{0}}{\omega d^{2}} \quad\left(2 \mu_{i} V_{0} / \omega d^{2}<1\right) \\
\Delta \varepsilon^{\prime \prime}(\omega)=\frac{q_{i}^{0} \mu_{i}}{\omega \varepsilon_{0}} \quad\left(2 \mu_{i} V_{0} / \omega d^{2}>1\right)
\end{array}\right.
$$

where $\Delta \varepsilon^{\prime \prime}$ and $\Delta \varepsilon^{\prime}$ are the changes of the complex permittivity in imaginary part and real part, respectively, $q_{i}^{0}$ is a constant charge density that is related to the nature of the electrode and dielectric liquid, $\mu_{i}$ is the mobility of the fast charge carriers and $V_{0}$ is the applied voltage between the two metal electrodes.

As seen from equation (24), the drift of these fast charge carriers can only contribute to the imaginary part of the complex permittivity. If those fast charge carriers can travel to the opposite electrode in a field cycle, $\Delta \varepsilon^{\prime \prime}(\omega)$ decreases with the frequency with a slope of -1 in a log-log scale, which is consistent with the experimental result [43]. Here, the polarization under the condition that these fast charge carriers are fast enough and can reach the opposite electrode in a cycle will be studied.

As discussed in our previous work, it is easy to reach a conclusion that the density of those fast charge carriers is $q_{i}^{0}$ everywhere [43]. If the slow charge carriers present between the two metal electrodes, the internal field is distorted. The field distribution in mineral oil under a low field $(2 \mathrm{~V} / \mathrm{mm})$ has been simulated and our simulation results indicate that the field distortion is negligible and can still be approximated as an evenly distributed field [42]. Therefore, the charge density of those fast charge carriers $\rho_{i}(x, t)$ should not differ much from their equilibrium values:

$$
\rho_{i}(x, t)=q_{i}^{0}+\gamma(x, t)
$$

where $\gamma(x, t)$ is a term that is much smaller compared with $q_{i}^{0}$.

Assuming there are no recombination and dissociation of those fast charge carriers, the current induced by those fast 
charge carriers can be written as:

$$
j_{i}(x, t)=\rho_{i}(x, t) \mu_{i} E(x, t)-D_{i} \frac{\partial \rho_{i}(x, t)}{\partial t}
$$

where, $\mu_{i}$ and $D_{i}$ is the mobility and the diffusion coefficient of the fast charge carriers. In our previous work, a new parameter, the ratio of the conductivity contributed by the motion of fast charge carriers over the total conductivity, has been defined. This ratio $\alpha$ has been defined as [42]

$$
\alpha=\frac{\sigma_{i}}{\sigma}
$$

If the electric field in mineral oil is homogeneous, the following expression can be obtained,

$$
\sigma_{i}=q_{i}^{0} \mu_{i}
$$

The ratios for three different kinds of mineral oil have been calculated. The maximum value of this ratio was 0.95 in our simulation. The mobility of those fast charge carriers has been estimated to be higher than $7.9 \times 10^{-5} \mathrm{~m}^{2} / \mathrm{s} / \mathrm{V}$ [43]. The conductivity of the mineral oil measured according to the procedures defined in IEC 61620 is $5.2 \times 10^{-11} \mathrm{~S} / \mathrm{m}$ [43].Thus the charge density carried by the fast charge carriers is smaller than $6.2 \times 10^{-7} \mathrm{C} / \mathrm{m}^{3}$. Assuming the mobility of slow charge carriers is $1 \times 10^{-8} \mathrm{~m}^{2} / \mathrm{s} / \mathrm{V}$, which is a typical value for the ionic mobility in mineral oil [45-46], the charge density carried by the slow charge carriers is $2.6 \times 10^{-4} \mathrm{C} / \mathrm{m}^{3}$. The charge density of the slow charge carriers is much more than that of the fast charge carriers. Thus, the internal field is mainly determined by the distribution of the slow charge carriers and can be denoted as

$$
E(x, t)=e^{i \omega t} E_{a} \frac{\cosh \left(k_{\omega} x\right)+i \omega \tau \cosh \left(k_{\omega} d\right)}{M}
$$

If $\gamma(x, t)$ is far smaller than $q_{i}^{0}$, the current due to the motion of these fast charge carriers becomes

$$
j_{i}(x, t)=q_{i}^{0} \mu_{i} E(x, t)-D_{i} \frac{\partial \rho_{i}(x, t)}{\partial x}
$$

With conservation of electric charge,

$$
\frac{\partial \rho_{i}(x, t)}{\partial t}+\frac{\partial j_{i}(x, t)}{\partial x}=0
$$

Employing equation (31), equation (30) becomes

$$
-\frac{\partial \rho_{i}}{\partial t}=q_{i}^{0} \mu_{i} \frac{\partial E(x, t)}{\partial x}-D_{i} \frac{\partial^{2} \rho_{i}}{\partial x^{2}}
$$

In steady state, the solution for the charge density should has the form:

$$
\rho_{i}(x, t)=\rho_{i \omega}(x) e^{i \omega t}
$$

Similarly, the current density due to the motion of these fast charge carriers can be denoted as

$$
j_{i}(x, t)=j_{i \omega}(x) e^{i \omega t}
$$

By substituting Eq. (33) into Eq. (32), we obtain:

$$
-B^{2} \rho_{i \omega}(x)=\frac{\sigma_{i}}{D_{i}} \frac{\partial E_{\omega}(x)}{\partial x}-\frac{d^{2} \rho_{i \omega}(x)}{d x^{2}}
$$

with

$$
\frac{i \omega}{D_{i}}=B^{2}
$$

where, $B$ is a frequency dependent parameter.

Employing Eq. (29), Eq. (35a) becomes

$$
-B^{2} \rho_{i \omega}(x)=A \sinh \left(k_{\omega} x\right)-\frac{d^{2} \rho_{i \omega}(x)}{d x^{2}}
$$

with

$$
A=\frac{\sigma_{i} E_{a} k_{\omega}}{\frac{\sinh \left(k_{\omega} d\right)}{k_{\omega} d}+i \omega \tau \cosh \left(k_{\omega} d\right)} \times \frac{1}{D_{i}}
$$

where, $A$ is a frequency dependent parameter.

Solution of equation (37) is

$$
\rho_{i \omega}(x)=-\frac{A \sinh \left(k_{\omega} x\right)}{B^{2}-k_{\omega}^{2}}+C_{1} \exp (B x)+C_{2} \exp (-B x)
$$

Here, we assume the charge injection can only be affected by the electric field at the two metal electrodes, $x= \pm d$, the current should follow

$$
\begin{gathered}
j_{i}(d, t)=\sigma_{i} E(d, t) \\
j_{i}(-d, t)=\sigma_{i} E(-d, t)
\end{gathered}
$$

By substituting equations (39a) and (39b) in equation (38) and solving for $C_{1}$ and $C_{2}$ gives

$$
C_{1}=\frac{q_{i}^{0}-\frac{P \sinh (B d)}{2}+P \exp (B d)}{\cosh (B d)}
$$

$$
C_{2}=\frac{q_{i}^{0}-\frac{P \sinh (B d)}{2}-P \exp (-B d)}{\cosh (B d)}
$$

with

$$
P=\frac{2}{\left(B^{2}-k_{\omega}^{2}\right) \sinh (B d)} \frac{\sigma_{i} E_{a} k_{\omega}}{\frac{1}{k_{\omega} d}+i \omega \tau \operatorname{coth}\left(k_{\omega} d\right)} \times \frac{1}{D_{i}}
$$

where, $P$ is a frequency dependent parameter. 
Therefore, the charge density of the fast charge carriers becomes

$$
\begin{aligned}
\rho_{i \omega}(x) & =-\frac{A \sinh \left(k_{\omega} x\right)}{B^{2}-k_{\omega}^{2}} \\
& +\frac{q_{i}^{0}-\frac{P \sinh (B d)}{2}+P \exp (B d)}{\cosh (B d)} \exp (B x) \\
& +\frac{q_{i}^{0}-\frac{P \sinh (B d)}{2}-P \exp (-B d)}{\cosh (B d)} \exp (-B x)
\end{aligned}
$$

The current induced in the external circuit by the motion of these fast charge carriers in the mineral oil is the average value of the internal current between the two metal electrodes. The current density in the external circuit can be denoted as

$$
\begin{aligned}
\frac{1}{2 d} \int_{-d}^{d} j_{i \omega}(x) d x & =\frac{1}{2 d} \int_{-d}^{d}\left[q_{i}^{0} \mu_{i} E_{\omega}(x)-D_{i} \frac{\partial \rho_{i \omega}(x)}{\partial x}\right] d x \\
& =\sigma_{i} E_{a}
\end{aligned}
$$

Thus, even under a non-homogenous field, the current caused by the drift and diffusion of those fast charge carriers can still only contribute to the imaginary part of the complex permittivity.

Employing equation (43), Coelho's space charge polarization theory should be modified as

$$
\varepsilon(\omega)=\varepsilon_{0} \varepsilon_{r}\left[\frac{1+i \omega \tau^{\prime}}{i \omega \tau^{\prime}+\tanh \left(k_{\omega}^{\prime} d\right) / k_{\omega}^{\prime} d}\right]-i \frac{\sigma_{i}}{\omega}
$$

with

$$
\left\{\begin{array}{l}
\tau^{\prime}=\varepsilon_{0} \varepsilon_{r} /\left(\sigma-\sigma_{i}\right) \\
k_{\omega}^{\prime 2}=\frac{1}{D \tau^{\prime}}\left(1+i \omega \tau^{\prime}\right)
\end{array}\right.
$$

It is easy to note that equations (44a) and (44b) coincide with equations (5) and (6) of [44]. Here, equation (44a) will be referred as the modified Coelho model.

\section{COMPUTER BASED POLARIZATION MODEL}

The computer based polarization model has been well described in our previous publications and thus only a brief introduction will be given here [42-43]. Assuming the slow charge carriers are distributed evenly between the two electrodes and two types of charge carriers that have the same properties except for their polarity are in the mineral oil, the density of positive charge carriers and negative charge carriers can be written as:

$$
n_{+}=n_{-}=n_{0}=\left(\sigma-\sigma_{i}\right) / q\left(\mu_{+}+\mu_{-}\right)
$$

The electric field is subject to the Poisson equation

$$
\partial E(x, t) / \partial x=q\left[n_{+}(x, t)-n_{-}(x, t)\right] / \varepsilon_{0} \varepsilon_{r}
$$

If the electric potential between the two electrodes is known as $V(t)$ and the distance between the two electrodes is $l=2 d$, the electric field should obey the following equation

$$
V(t)=\int_{0}^{l} E(x, t) d x
$$

If assuming charge carriers are dissociated from ionic pairs, the relationship between ionic pairs and free charge carriers can be described as [47]

$$
-\frac{d c}{d t}=\frac{d n_{+}}{d t}=\frac{d n_{-}}{d t}=K_{d} c-K_{r} n_{+} n_{-}
$$

where $c$ is concentration of ionic pairs, $K_{d}$ is the dissociation rate and $K_{r}$ is the recombination rate. The recombination rate can written as [41]

$$
K_{r}=q\left(\mu_{+}+\mu_{-}\right) / \varepsilon_{0} \varepsilon_{r}
$$

Since the mineral oil can be treated as weak electrolyte, therefore, the density of ionic pairs is much larger than the density of free charge carriers. Therefore, the density of ionic pairs would not change much from its original value. Here, equation (49) can be rewritten as

$$
-\frac{d c}{d t}=\frac{d n_{+}}{d t}=\frac{d n_{-}}{d t}=K_{d} c-K_{r} n_{+} n_{-}=K_{r} n_{0}^{2}-K_{r} n_{+} n_{-}
$$

When the flow of liquid can be ignored, the density of positive and negative charge carriers can be denoted as

$$
\begin{aligned}
\frac{d n_{+}(x, t)}{d t} & =K_{r} n_{0}^{2}-K_{r} n_{+}(x, t) n_{-}(x, t)+D_{+} \frac{\partial^{2} n_{+}(x, t)}{\partial x^{2}} \\
& -\mu_{+} \partial\left[n_{+}(x, t) E(x, t)\right] / \partial x \\
\frac{d n_{-}(x, t)}{d t} & =K_{r} n_{0}^{2}-K_{r} n_{+}(x, t) n_{-}(x, t)+D_{-} \frac{\partial^{2} n_{-}(x, t)}{\partial x^{2}} \\
& +\mu_{-} \partial\left[n_{-}(x, t) E(x, t)\right] / \partial x
\end{aligned}
$$

Assuming all slow charge carriers will be blocked at the interface, the current flow through the circuit can be calculated from the change of the induced charge at the electrode which is attracted by the space charge in the bulk. This induced charge due to space charge in the bulk can be expressed as

$$
Q(t)=\frac{q}{l} \int_{0}^{l} x\left[n_{+}(x, t)-n_{-}(x, t)\right] d x
$$

When the external electric potential that applied upon the two electrodes is a simple sinusoidal voltage $V(t)=V_{0} \sin (\omega t)$, the relative dielectric permittivity involves electrode polarization can be written as:

$$
\left\{\begin{array}{c}
\varepsilon^{\prime \prime}(\omega)=2 I_{\text {real }} l /\left(\varepsilon_{0} \omega V_{0} S\right)+\sigma_{i} / \omega \varepsilon_{0} \\
\varepsilon^{\prime}(\omega)=2 I_{\text {imag }} l /\left(\varepsilon_{0} \omega V_{0} S\right)+\varepsilon_{\infty}
\end{array}\right.
$$

with

$$
\left\{\begin{array}{l}
I_{\text {real }}=f \int_{0}^{1 / f}(d Q(t) / d t) S \sin (\omega t) d t \\
I_{\text {imag }}=f \int_{0}^{1 / f}(d Q(t) / d t) S \cos (\omega t) d t
\end{array}\right.
$$


where, $f$ is the frequency, $S$ is the surface area of the electrode, $\omega$ is the angular frequency. $\varepsilon_{\infty}$ is the relative permittivity at high frequency. $I_{\text {real }}$ is the current that has the same phase with the electric potential, while $I_{\text {imag }}$ is the current that leads the applied ac voltage by an angle of $90^{\circ}$. These equations can be easily obtained by integrating the current flow through a pure ohmic sample that has a constant conductivity and permittivity [19].

\section{COMPARATION OF THE MODIFIED SPACE CHARGE POLARIZATION MODEL AND THE EXPERIMENTAL RESULT}

In this paper, the frequency responses of three different types of mineral oil: the fresh oil, the lightly aged oil and the heavily aged oil have been studied and analysed.

The fresh oil was Shell ZX-I mineral oil provided by Shell Company. The lightly aged oil was obtained from a HVDC transformer, which has been in service for about 10 years. The heavily aged oil was taken from a failed HVDC transformer, which has been aged over 50 years. The water contents in these three oils were measured by Karl-Fisher titration technique and they are $4 \mathrm{ppm}, 12 \mathrm{ppm}$ and $24 \mathrm{ppm}$ respectively. Their viscosities were measured using Physica Rheolab $\mathrm{MCl}$ instrument fitted with a concentric cylinder test cell and their viscosity at $30{ }^{\circ} \mathrm{C}$ are $10.3 \mathrm{mPa} \cdot \mathrm{s}, 9.5 \mathrm{mPa} \cdot \mathrm{s}$ and $15.2 \mathrm{mPa} \cdot \mathrm{s}$ respectively. Their DC conductivity at $30{ }^{\circ} \mathrm{C}$, which were measured based on IEC 61620, are $0.31 \mathrm{pS} / \mathrm{m}, 7.6 \mathrm{pS} / \mathrm{m}$ and $150 \mathrm{pS} / \mathrm{m}$. Before each measurement, the oil sample has been put into a vacuum chamber to remove the dissolved gas.

The dielectric spectroscopy tests were performed according to IEC 60247. The frequency-domain measurements have been carried out using the Solartron 1296 dielectric interface linked to the model 1260A impedance/-gain phase analyser. The schematic diagram for the test cell is illustrated in Fig. 1. A parallel cup-plate arrangement of two metal electrodes with a diameter of $33 \mathrm{~mm}$ has been used here. The potential difference between these two metal electrodes is $1 \mathrm{~V}$ and a PTFE spacer with a thickness of $0.5 \mathrm{~mm}$ has been inserted between these two electrodes. The electrode system was placed in a heating chamber. The experiments have been performed at four different temperatures $(25,50,75$ and 90 ${ }^{\circ} \mathrm{C}$ ). To ensure a good contact between oil sample and the metal electrodes, the surface of the upper electrode needs to be submerged in oil. About $0.5 \mathrm{~mL}$ mineral oil is used in each measurement. The test cell filled with oil was maintained at the desired temperature for at least half an hour before each measurement. The frequency range for the test is $100 \mathrm{~Hz}-$ $0.01 \mathrm{~Hz}$. The experimental results have been recorded by a computer through an IEEE 488 cable.

In our simulation, the total conductivity is calculated from the imaginary part of the complex permittivity obtained experimentally using $\sigma=\omega \varepsilon_{0} \varepsilon^{\prime \prime}(\omega)$. The relative permittivity $\varepsilon_{r}$ is taken directly from the real part of the complex permittivity at $100 \mathrm{~Hz}$ measured in the experiments. The mobility of these charge carriers is assumed to be proportional to the reciprocal of the viscosity and the mobility of the charge carriers in the heavily aged oil is assumed to be $1 \times 10^{-9} \mathrm{~m}^{2} / \mathrm{s} / \mathrm{V}$. The temperature dependence of the viscosity of the oil samples can be found in our previous publication [42].

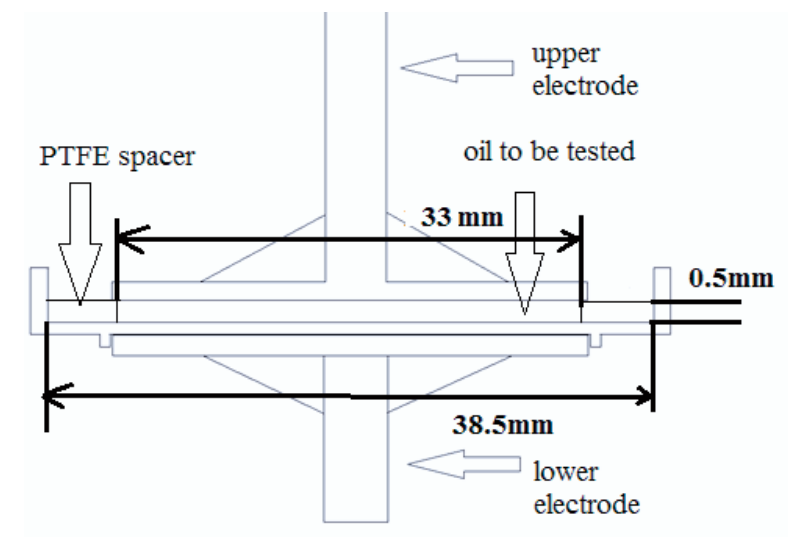

Figure 1. Schematic diagram for the test cell used in dielectric spectroscopy measurement.

The computer based simulation has been discussed in our previous publication and the simulated results are involved here for comparison [43]. Figures 1-3 show the experimental result and the simulation result of the frequency dependence of the real part of the complex permittivity at different temperature. The star marker, the triangle marker, the square marker and the circular marker stand for the value of the real part of the complex permittivity obtained from the experiment at $90,75,50$ and $25^{\circ} \mathrm{C}$, respectively. The red, blue, green and pink solid (dashed) lines represent the values obtained from modified Coelho model (computer based simulation) at 90, 75, 50 and $25^{\circ} \mathrm{C}$, respectively.

The characteristics of the frequency response of insulating oils shown in this paper have been widely observed by other researchers [13-18]. Although the mineral oils tested here have gone through different types of stresses and their original conditions may be different slightly from each other, the frequency responses of these three mineral oils can give an overall picture of the effect of oil conditions when they are fresh, in service for several years and heavily aged that needs to be replaced.

As seen from Figures 2-4, the real parts of the relative complex permittivity of the mineral oil are around 2.1-2.4 in frequency ranges from $1 \mathrm{~Hz}$ to $100 \mathrm{~Hz}$ and they does not change much regardless of the AC conductivity. As pointed out by Sawada, the permittivity in this range studied is mainly contributed by electronic, atomic and dipole polarizations and these polarizations have been fully polarized so that the real part of the complex permittivity seems to be constant [31]. The increase of the real part of the complex permittivity indicates space charge polarization can affect the frequency response in mineral oil when the frequency is below $1 \mathrm{~Hz}$. More charge carriers are generated in the aging of mineral oil. Because there are more charge carriers participating the ionic drift and diffusion process, the space charge polarization becomes more significant and a higher real part of the complex permittivity can be observed in the aged mineral oil. Also, the curves of the real part of the complex permittivity 
shift towards high frequency when the temperature increases, which is due to that the density of the charge carriers and the mobility increase with the temperature.

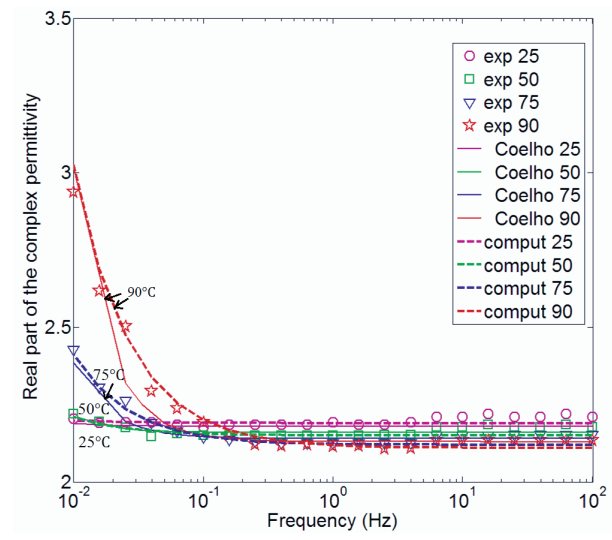

Figure 2. Theoretical and experimental results of the real part of the complex permittivity of the fresh oil. Simulating results and experimental results from [43] are involved here for comparison.

As seen from Figures 2-4, the modified Coelho model can fit the real part of the complex permittivity. The real part of the complex permittivity does not change much in a frequency range of 1 to $100 \mathrm{~Hz}$ and shows increase when the frequency decreases further. However, the computer based method surly provides a better fit. Because the mineral oil can be treated as weak electrolyte, the quantity of charge carriers that dissociated from ionic pairs should not be simply ignored especially when the frequency is low. Because the dissociation can produce extra charge carriers, the space charge polarization can be enhanced by the motion of these newly generated charge carriers. Consequently, the computer-based calculated value of the real part of the complex permittivity increases faster. Therefore, in order to get a more accurate experimental fit, the computer based method is recommended.

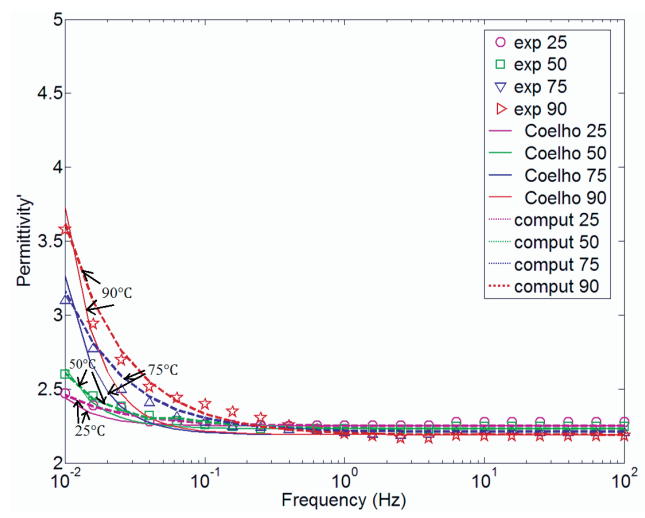

Figure 3. Theoretical and experimental results of the real part of the complex permittivity of the lightly aged oil. Simulating results and experimental results from [43] are involved here for comparison.

Figures 5-7 show the experimental result and the simulation result of the frequency dependence of the imaginary part of the complex permittivity at different temperature. The star marker, the triangle marker, the square marker and the circular marker stand for the value of the imaginary part of the complex permittivity obtained from the experiment at 90, 75, 50 and $25^{\circ} \mathrm{C}$, respectively. The red, blue, green and pink solid lines represent the values obtained from modified Coelho model at $90,75,50$ and $25^{\circ} \mathrm{C}$, respectively. The difference between the values of the imaginary part of the complex permittivity obtained from computer based simulation [43] and the modified Coelho model is negligible, thus, only the values that calculated from the computer based simulation are shown in Figures 5-7.

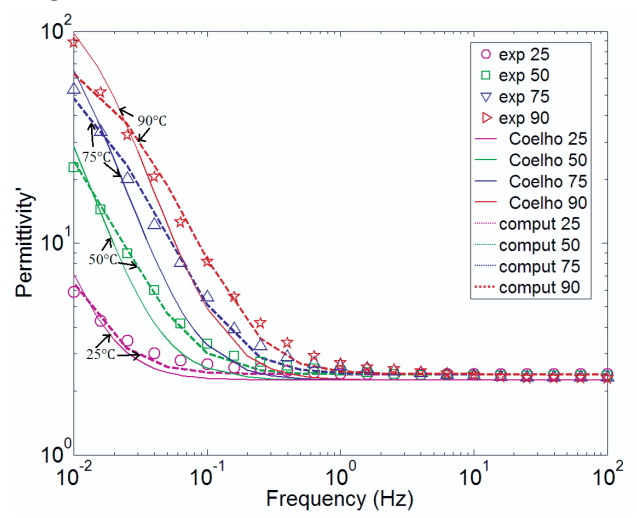

Figure 4. Theoretical and experimental results of the real part of the complex permittivity of the heavily aged oil. Simulating results and experimental results from Ref. [43] are involved here for comparison.

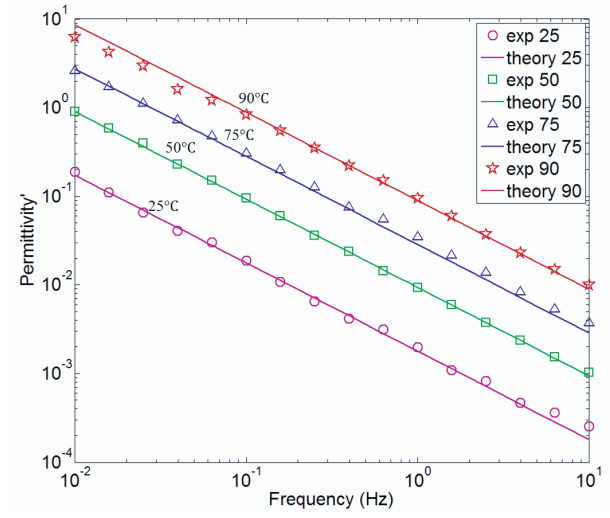

Figure 5. Theoretical and experimental results of the imaginary part of the complex permittivity of the fresh oil. Experimental results from [43] are involved here for comparison.

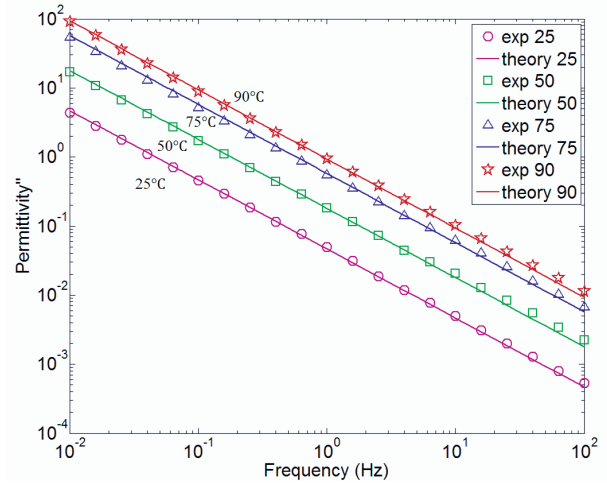

Figure 6. Theoretical and experimental results of the imaginary part of the complex permittivity of the lightly aged oil. Experimental results from [43] are involved here for comparison.

As seen from Figures 5-7, the fresh oil has the lowest dielectric loss, the lightly aged oil has a medium dielectric loss and the heavily aged oil has the highest dielectric loss. The dielectric loss of the mineral oil increases with aging [1-8]. As the conductivity of mineral oil also increases with the temperature, the curves of the imaginary part of the complex permittivity shift towards high frequency when the temperature increases. The imaginary part of the complex 
permittivity decreases linearly with the frequency in a slope of -1 in log-log scale regardless the conductivity indicates a nearconstant conductivity of the mineral oil in the frequency range studied $(100-0.01 \mathrm{~Hz})$. The imaginary part of the complex permittivity that calculated from the computer based simulation can reach a peak and then start to decrease [24-35], which is against the experimental result. This inconsistence has been attributed to a low density of highly mobile charge carriers which can be generated in the vicinity of the electrode [37]. As observed from Figures 5-7, good fittings between observed and calculated values are achieved for the frequencydependent curves of the complex permittivity. It seems if there are fast charge carriers in the mineral oil, both the real and imaginary part of the complex permittivity can be fitted.

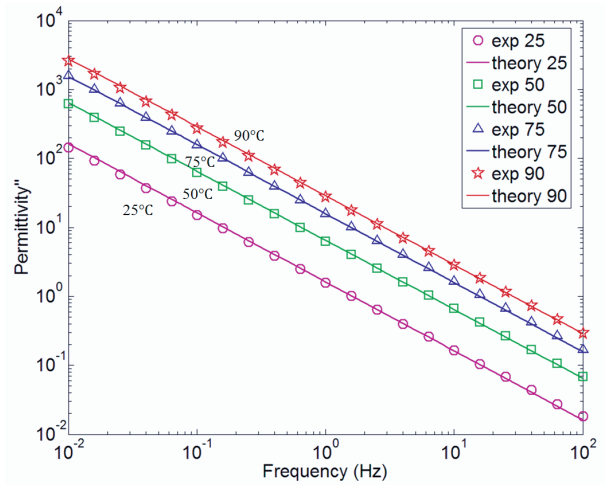

Figure 7. Theoretical and experimental results of the imaginary part of the complex permittivity of the heavily aged oil. Experimental results from [43] are involved here for comparison.

In our previous work, the ratio of conductivity contributed by the motion of the fast charge carriers over the total conductivity, $\alpha$, has been well studied and we have found that this parameter increases with temperature and aging [42-44].

As seen from Table, the value of $1-\alpha$ obtained from two different calculating methods have a good consistence. The density of the fast charge carriers increases with temperature and aging. It seems this parameter can be used to assess the dielectric properties of the mineral oil. A high value of $\alpha$ means the oil has been seriously aged. Since the calculation based on the modified Coelho model is much easier and faster than the computer based method, equation (44) can be used in daily measurement analysis. If the ratio $\alpha$ of the mineral oil is known, the conductivity contributed by the dissociated charge carriers can reveal the condition of the mineral oil as this conductivity depends on the properties of the molecules and the ions. It is well accepted that the dissociation of gas and moisture can increase the conductivity of the mineral oil. If the hydrocarbon molecules in the oil have not suffered from chemical degradation, the dielectric properties of the oil can be significantly improved by removing the dissolved gas and the moisture. However, if the oil is heavily aged and the hydrocarbon molecules have been heavily degraded, it is be better replacing the oil rather than going through a reconditioning process. The conductivity contributed by the motion of the slow charge carriers, which is going to referred as dissociated conductivity in the following sections, can be denoted as

$$
\sigma_{d}=(1-\alpha) \sigma
$$

Table 1. Value of 1- $\alpha$ for three different oils using computer based method.

\begin{tabular}{c|c|c|c|c|c}
\hline \multicolumn{2}{c|}{} & $25^{\circ} \mathrm{C}$ & $50{ }^{\circ} \mathrm{C}$ & $75^{\circ} \mathrm{C}$ & $90^{\circ} \mathrm{C}$ \\
\hline Fresh oil & $\begin{array}{c}\text { Computer based } \\
\text { method }\end{array}$ & 0.90 & 0.70 & 0.60 & 0.45 \\
\cline { 2 - 6 } & $\begin{array}{c}\text { Modified } \\
\text { Coelho model }\end{array}$ & 0.80 & 0.45 & 0.40 & 0.38 \\
\hline $\begin{array}{c}\text { Lightly } \\
\text { aged oil }\end{array}$ & $\begin{array}{c}\text { Computer based } \\
\text { method }\end{array}$ & 0.40 & 0.15 & 0.10 & 0.065 \\
\cline { 2 - 6 } & $\begin{array}{c}\text { Modified } \\
\text { Coelho model }\end{array}$ & 0.30 & 0.11 & 0.055 & 0.050 \\
\hline $\begin{array}{c}\text { Heavily } \\
\text { aged oil }\end{array}$ & $\begin{array}{c}\text { Computer based } \\
\text { method }\end{array}$ & 0.14 & 0.11 & 0.080 & 0.070 \\
\cline { 2 - 6 } & $\begin{array}{c}\text { Modified } \\
\text { Coelho model }\end{array}$ & 0.11 & 0.095 & 0.090 & 0.080 \\
\hline
\end{tabular}

The temperature dependence of the dissociated conductivities of the mineral oils are shown in Table 2. As seen from Table 2, the dissociated conductivity increase with the temperature and the oil aging period. Although the total conductivity of lightly aged oil is about 20 times higher than that of the fresh oil, the dissociated conductivity of the lightly aged oil is about 11 times higher than that of the fresh oil at 25 ${ }^{\circ} \mathrm{C}$ and 1.6 times at $90{ }^{\circ} \mathrm{C}$, which suggested that there might be some highly dissolvable impurities in the lightly aged oil. As the temperature increases, the density of the charge carriers dissociated from these highly dissolvable impurities do not change much. Consequently, the difference between the dissociated conductivity of the lightly aged oil and the fresh oil becomes smaller with the increase of the temperature. The heavily aged oil, which has been aged for 50 years, the hydrocarbon molecules of this oil has been severely degraded and smaller molecules are created under electrical, thermal and chemical stresses [48-49]. Therefore, the heavily aged oil has the highest dissociated conductivity and it might not be worthy to have this oil reconditioned. However, how can the dissociated conductivity be related to the chemical degradation of the hydrocarbon molecules is still not very clear and more research is needed.

Table 2. Dissociated conductivity and total conductivity for mineral oil with different aging period.

\begin{tabular}{c|c|c|c|c|c}
\multicolumn{2}{c|}{ Conductivity $(\mathrm{pS} / \mathrm{m})$} & $25{ }^{\circ} \mathrm{C}$ & $50{ }^{\circ} \mathrm{C}$ & $75^{\circ} \mathrm{C}$ & $90{ }^{\circ} \mathrm{C}$ \\
\hline \multirow{2}{*}{ Fresh oil } & $\sigma_{d}$ & 0.09 & 0.37 & 0.96 & 1.6 \\
\cline { 2 - 6 } & $\sigma$ & 0.10 & 0.53 & 1.6 & 3.6 \\
\hline \multirow{2}{*}{$\begin{array}{c}\text { Lightly } \\
\text { aged oil }\end{array}$} & $\sigma_{d}$ & 1.1 & 1.5 & 3.2 & 3.5 \\
\cline { 2 - 6 } & $\sigma$ & 2.6 & 10 & 32 & 51 \\
\hline \multirow{2}{*}{$\begin{array}{l}\text { Heavily } \\
\text { aged oil }\end{array}$} & $\sigma_{d}$ & 13 & 40 & 70 & 110 \\
\cline { 2 - 6 } & $\sigma$ & 90 & 360 & 880 & 1500 \\
\hline
\end{tabular}

It is worth mentioning that the modified Coelho theory is developed based on the assumption that the internal field distortion is not serious. When this frequency-domain measurement is carried out under high electric field, more charge carriers will be created and the field distortion can be significant. Thus, this modified model might not be valid if the field is high. Also, as the frequency goes low, almost all the slow charge carriers are able to travel to a region that is close to the electrode. If assuming a mobility of $1 \times 10^{-9} \mathrm{~m}^{2} / \mathrm{s} / \mathrm{V}$, the 
frequency at which all the dissociated charge carriers can travel to the electrode can be calculated as:

$$
f=\frac{2 \mu E}{2 \pi l}=\frac{2 \times 10^{-9} \times 2 \times 10^{3}}{2 \pi \times 5 \times 10^{-4}}=1.27 \times 10^{-3}(\mathrm{~Hz})
$$

When the frequency is below $0.001 \mathrm{~Hz}$, all these charge carriers can reach the electrodes resulting in an uneven charge distribution. Under this condition, equations (7a) and (7b) are no longer valid along with the modified Coelho model. To sum up, this modified model cannot be used to explain the dielectric behaviour of mineral oil under a very low frequency or a very high electric field.

If these charge carriers do exist, the electric conduction under DC field can be affected by the motion of these fast charge carriers. When the electric field is removed, the dissociated charge carriers will remain in the oil, whilst the fast charge carriers will be extracted within a very short of time. Therefore, the depolarization current is mainly determined by the dissociated charge carriers. In our previous work, the ratio of conductivity contributed by the motion of the fast charge carriers over the total conductivity calculated based on the time domain measurement is consistent with that obtained from the dielectric spectroscopy measurement [50]. However, the nature of these fast charge carriers is not very clear and more research is needed.

\section{CONCLUSION}

The present work is focused on the analysis of frequency response of the mineral oil. The dielectric loss of mineral oil increases with aging and temperature. The real part of the complex permittivity increases faster when the oil is aged, whilst the imaginary part of permittivity decrease with the frequency with a slope of -1 regardless of the conductivity. The motion of the fast charge carriers can only contribute to the imaginary part of the complex permittivity even under a nonhomogeneous field. The modified Coelho model can be used to explain the dielectric behaviour of the mineral oil. The ratio of the conductivity contributed by the motion of the fast charge carriers over the total conductivity obtained from the modified Coelho model consists with that obtained from the computer based simulation. A new parameter, the dissociated conductivity, may be used as one of the indicators of the oil aging. For daily test, the modified Coelho model can be used for its simplicity. However, the nature of these fast charge carriers is still not well understood and more researches are needed.

\section{ACKNOWLEDGMENT}

The authors are grateful to the National Grid for their financial support.

\section{REFERENCES}

[1] R. Bartnikas, Engineering Dielectrics Vol. III, Electrical Insulating Liquids, ASTM, 1994.

[2] Y. Jing, I. V. Timoshkin, M. P. Wilson, M. J. Given, S. J. Macgregor, T. Wang and J. M. Lehr, "Dielectric Properties of Natural Ester, Synthetic Ester Midel 7131 and Mineral Oil Diala", IEEE Trans. Dielectr. Electr. Insul., Vol. 21, pp. 644 - 652, 2014.

[3] S. Singha, R. Asano, G. Frimpong, C. C. Claiborne, D. Cherry, "Comparative Aging Characteristics between a High Oleic Natural Ester Dielectric Liquid and Mineral Oil”, IEEE Trans. Dielectr. Electr. Insul., Vol. 21, pp. 149-158, 2014.

[4] R. Bartnikas, "Dielectric Loss in Insulating Liquids", IEEE Trans. Electr. Insul., Vol. 2, pp. 33-54, 1967.
[5] A. A. S. Akmal, H. Borsi, E. Gockenbach and V. Wasserberg, "Dielectric Behavior Insulating Liquid at Very Low Frequency”, IEEE Trans. Dielectr. Electr. Insul., Vol.13, pp. 532-538, 2006.

[6] W. S. Zaengl, "Dielectric Spectroscopy in Time and Frequency Domain for HV Power Equipment, Part I: Theoretical Considerations.", IEEE Electr. Insul. Mag., Vol. 19, No. 5, pp. 5-19, 2003.

[7] C. T. Dervos, C. D. Paraskevas and P. Skafidas, "Dielectric Characterization of Power Transformer Oils as a Diagnostic Life Prediction Method", IEEE Electr. Insul. Mag., Vol. 21,pp. 11-19, 2005

[8] T. K. Saha, "Review of Modern Diagnostic Techniques for Assessing Insulation Condition in Aged Transformers", IEEE Trans. Dielectr. Electr. Insul., Vol. 10, pp. 903-917, 2003.

[9] C. D. Paraskevas, P. Vassiliou and C. T. Dervos, "Temperature Dependent Dielectric Spectroscopy in Frequency Domain of High-Voltage Transformer Oils Compared to Physicochemical Results", IEEE Trans. Dielectr. Electr. Insul., Vol. 13, pp. 281-284, 2005.

[10] I. Adamczewski. Ionization, Conductivity and Breakdown in Dielectric Liquids, Taylor \& Francis, 1969.

[11] D. Linhjell, O. L. Hestad, U. Gafvert, and L. E. Lundgaard, "Dielectric Response of Oil-impregnated Cellulose from $0.1 \mathrm{mHz}$ to $3 \mathrm{MHz}$,, IEEE Int'l. Conf. Dielectr. Liquids (ICDL), pp. 277-280, 2005.

[12] L. J. Yang, S. M. Gubanski, Y. V. Serdyuk and J. Schiessling, "Dielectric properties of transformer oils for HVDC applications", IEEE Trans. Dielectr. Electr. Insul., Vol. 19, pp. 1926-1933, 2012.

[13] A. Haidar, J. C. Fothergill and L. A. Dissado, "Anomalous Dielectric Response of Very Small Quantities of Virgin, Aged and Failed Silicone Oil", IEEE Trans. Dielectr. Electr. Insul., Vol. 10, pp. 336-342, 2003.

[14] C. T. Duy, A. Denat, O. Lesaint, N. Bonifaci, and Y. Bertrand, "Moisture and temperature effects on conduction and losses in modified rape-seed insulating oil", IEEE Conf. Electr. Insul. Dielectr. Phenomena (CEIDP), pp. 647-650, 2007.

[15] I. L. Hosier, A. Guushaa, E. W. Westenbrink, C. Rogers, A. S. Vaughan and S. G. Swingler, "Aging of Biodegradable Oils and Assessment of their Suitability for High Voltage Applications", IEEE Trans. Dielectr. Electr. Insul., Vol. 18, pp. 728-737, 2011.

[16] B. S. H. M. S. Y. Matharage, M. A. R. M. Fernando, M. A. A. P. Bandara, G. A. Jayantha and C. S. Kalpage, "Performance of Coconut Oil as an Alternative Transformer Liquid Insulation", IEEE Trans. Dielectr. Electr. Insul., Vol. 20, pp. 887-898, 2013.

[17] J. Ulrych, M. Svoboda, R. Polansky, J. Pihera, "Dielectric Analysis of Vegetable and Mineral Oils", IEEE Int'l. Conf. Dielectr. Liquids (ICDL), pp. 14, 2014.

[18] K. Bandara, C. Ekanayake and T. K. Saha, "Comparative Study for Understanding the Behaviour of Natural Ester with Mineral oil as a Transformer Insulating Liquid", IEEE Conf. Electr. Insul. Dielectr. Phenomena (CEIDP), pp. 792-795, 2014.

[19] F. Kremer and A. Schonhals, Broadband Dielectric Spectroscopy, Springer, 2003.

[20] G Jaffé, “Theory of Conductivity of Semiconductors", Phys. Rev., Vol. 85, pp. 354-363, 1952.

[21] H. C. Chang and G. Jaffé, "Polarization in Electrolytic Solutions. Part I. Theory”, J. Chem. Phys. Vol. 20, pp. 1071-1087, 1952.

[22] G. Jaffé and C. Z. LeMay, "On Polarization in Liquid Dielectrics", J. Chem. Phys., Vol. 21, 920-928, 1953.

[23] G. Jaffé and J. A. Rider, "Polarization in Electrolytic Solutions. Part II. Measurements", J. Chem. Phys., Vol. 20, pp. 1077-1087, 1952.

[24] J. R. Macdonald, "Theory of ac Space-Charge Polarization Effects in Photoconductors, Semiconductors, and Electrolytes", Phys. Rev., Vol. 92, pp. 4-17, 1953.

[25] F. C. M. Freire, G. Barbero, and M. Scalerandi, "Electrical Impedance for an Electrolytic Cell", Phys. Rev, E, Vol. 73, 051202, 2006.

[26] P. W. M. Jacobs and J. N. Maycock, "Polarization Effects in the Ionic Conductivity of Alkali Halide Crystals. I. AC Capacity", J. Chem. Phys., Vol. 39, pp. 757-762, 1963.

[27] F. Stern and C. Weaver, "Dispersion of Dielectric Permittivity due to Space Charge Polarization", J. Phys. C., Vol. 3, pp. 1736-1746, 1970.

[28] R. J. Klein, S. Zhang, S. Dou, B. H. Jones, R. H. Colby, and J. Runt, "Modeling Electrode Polarization in Dielectric Spectroscopy: Ion Mobility and Mobile Ion Concentration of Single-Ion Polymer Electrolytes", J. Chem. Phys., Vol. 124, 144903, 2006.

[29] A. D. Hollingsworth and D. A. Saville, "A Broad Frequency Range Dielectric Spectrometer for Colloidal Suspensions: Cell Design, Calibration, and Validation", J. Colloid Interface Sci., Vol. 257, pp. 65-76, 2003. 
[30] G. Barbero, "Influence of Adsorption Phenomenon on the Impedance Spectroscopy of a Cell of Liquid", Phys. Rev. E, Vol. 71, 062201, 2005.

[31] A. Sawada, "Space-Charge Polarization of a Dilute Electrolytic Cell in the Presence of Diffuse Double Layers", J. Appl. Phys., Vol. 112, 044104, 2012.

[32] A. Sawada, "Internal Electric Fields of Electrolytic Solutions Induced by SpaceCharge Polarization", J. Appl. Phys., Vol. 100, 074103, 2006.

[33] A. Sawada, "Theory of Space-Charge Polarization for Determining Ionic Constants of Electrolytic Solutions", J. Chem. Phys., Vol. 126, 224515, 2007.

[34] A. Sawada, "Dielectric Process of Space-Charge Polarization for an Electrolytic Cell with Blocking Electrodes", J. Chem. Phys., Vol. 129, 064701, 2008.

[35] A. Sawada, "Modeling of Electrode Polarization for Electrolytic Cells with a Limited Ionic Adsorption", Phys. Rev E, Vol. 88, 032406, 2013

[36] R. Coelho, Physics of Dielectrics for the Engineer, Elsevier New York, 1979.

[37] D. G. Frood and T. J. Gallagher, "Space-Charge Dielectric Properties of Water and Aqueous Electrolytes", J. Mol. Liq., Vol. 69, pp. 183-200, 1996.

[38] R. Richert, A. Agapov and A. P. Sokolov, "Appearance of a Debye Process at the Conductivity Relaxation Frequency of a Viscous Liquid", J. Chem. Phys., Vol. 134, 104508, 2011.

[39] G. Power, M. Nagaraj, J. K. Vij, and G. P. Johari, "Debye Process and Dielectric State of an Alcohol in a Nonpolar Solvent", J. Chem. Phys., Vol. 134, 044525, 2011.

[40] B. A. Mazzeo and A. J. Flewitt, "Two- and Four-Electrode, Wide-Bandwidth, Dielectric Spectrometer for Conductive Liquids: Theory, Limitations, and Experiment", J. Appl. Phys., Vol. 102, 104106, 2007.

[41] A. Oleinikova, P. Sasisanker, and H. Weingartner "What Can Really Be Learned from Dielectric Spectroscopy of Protein Solutions? A Case Study of Ribonuclease A", J. Phys. Chem. B, Vol. 108, 8467, 2004.

[42] Y. Zhou, M. Hao, G. Chen, G. Wilson and P. Jarman, "Space Charge Polarization in Insulating Mineral Oil", IEEE Conf. Electr. Insul. Dielectr. Phenomena (CEIDP), Vol. 1, pp. 587-590, 2013,

[43] Y. Zhou, M. Hao, G. Chen, G. Wilson and P. Jarman, "Study of the Dielectric Response in Mineral Oil Using Frequency-Domain Measurement", J. Appl. Phys., Vol. 115, 124105, 2014.

[44] Y. Zhou, M. Hao, G. Chen, G. Wilson and P. Jarman, "A New Approach to Understanding the Frequency Response of Mineral Oil", ICDL, Vol. 1, 1-4, 2014

[45] L. Yang, M. S. Zadeh, J. Schiessling, O. Hjortstam, Y. V. Serdyuk and S. M. Gubanski, "Measurement of Ion Mobility in Transformer Oils for HVDC Applications", Int'l Conf. High Voltage Eng. (ICHVE), pp. 464-467, 2012

[46] O. Hjortstam, J. Schiessling, Y. V. Serdyuk and S. M. Gubanski, "Measurements of Ion Mobility in Transformer Oil: Evaluation in terms of Ion Drift", IEEE Conf. Electr. Insul. Dielectr. Phenomena (CEIDP), pp. 495-498, 2012.

[47] A. Castellanos, Electrohydrodynamics, Springer, Wien New York, 1998.

[48] R. Sanghi, "Chemistry Behind the Life of a Transformer", Resonance, Vol. 8, pp. 17-23, 2003.

[49] L. Zhonghua, Z. Jun, Y. Yi, M. Jun, W. Xinsheng, and T. Demin "Formation and Inhibition of Free-Radicals in Insulating Materials Aged by Electrical Field", IEEE Int'l. Conf. Properties Applications Dielectr. Materials (ICPADM), pp. 25-30 1997.

[50] Y. Zhou, M. Hao, G. Chen, G. Wilson and P. Jarman, " Study of the Depolarization Current in Mineral Oils", IEEE Int'l. Conf. Dielectr. Liquids (ICDL), pp. 1-4, 2014.

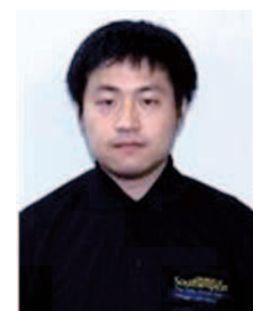

Yuan Zhou was born in China in 1987. He received his B.Eng. degree in electrical engineering from Xi'an Jiaotong University, China, in 2009. Since 2011, he has been a Ph.D. degree student in the University of Southampton, UK. His main research interests include electrical conduction and space charge in the oil impregnated pressboard.

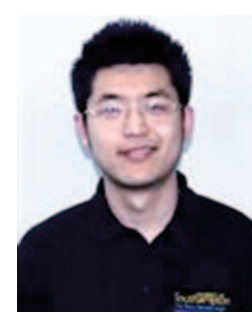

Miao Hao was born in China in 1987. He received his B.Eng. degree (2009) from Xi'an Jiaotong University, China and M.Sc. degree (2011) from the University of Southampton, UK in electrical engineering. Since 2011, he has been a Ph.D. degree student in the University of Southampton, UK. His main research interests include space charge and ageing mechanism in the oil impregnated pressboard insulation system for HVDC converter transformer.

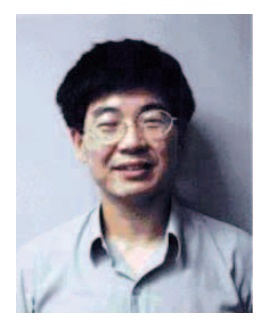

George Chen (SM'11) was born in China in 1961. He received the B.Eng. (1983) and M.Sc. (1986) degrees in electrical engineering from Xi'an Jiaotong University, China. After he obtained the Ph.D. degree (1990) from the University of Strathclyde, UK, on the work of permanent changes in electrical properties of irradiated low-density polyethylene, he joined the University of Southampton as postdoctoral research fellow and became a senior research fellow subsequently. In 1997 he was appointed as a research lecturer and promoted to a Reader in 2002. He is now the professor of high voltage engineering at the University of Southampton and a visiting professor of Xi' an Jiaotong University. Over the years, he has developed a wide range of interests in high voltage engineering and electrical properties of materials and published over 300 papers.He is active in the HVDC systems and involved with technical working groups in both IEEE and CIGRE.

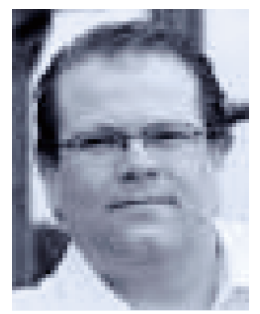

Gordon Wilson completed his chemistry degree at the University of Surrey in 1995, this was followed by a National Grid-sponsored Ph.D. degree in characterizing mineral transformer oil, also at the University of Surrey. He joined National Grid in 1999 as an oil chemist providing support to a team of transformer specialists. Since 2007 he has worked on transformer thermal ratings whilst retaining responsibility for transformer oil issues. Gordon is a member of the BSi National Committee for electrotechnical fluids, he is the UK regular member for CIGRE Study Committee on materials and he is a member of the IEEE Dielectrics and Electrical Insulation Society.

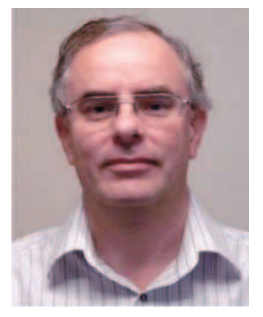

Paul Jarman was born in London on 27 September 1962, and graduated from Cambridge University in 1984 with an Honours degree in electrical science. He joined the Central Electricity Generating Board, Research Division, working on, amongst other projects, FRA testing of transformers. In 1990, he joined the National Grid as a transformer engineer, becoming head of transformers in 1998. Since 2001, Jarman has been National Grid's technical specialist for transformers now within the Asset Management group. Jarman is chairman of IEC TC14, the international committee for power transformer standards; is the UK regular member of CIGRE study committee A2 for transformers; and has recently been the convenor of a CIGRE group on transformer monitoring. He is a chartered electrical engineer and member of the IET. 